\title{
VALOR PREDICTIVO DE LA INTELIGENCIA EMOCIONAL RASGO EN LOS ESTADOS AFECTIVOS: UNA COMPARACIÓN DE GÉNERO
}

\author{
Ana Merchán-Clavellino \\ Departamento de Psicología, Universidad de Cádiz. \\ Instituto Universitario de Investigación para el Desarrollo Social Sostenible (INDESS), Universidad de Cádiz. \\ ana.merchan@uca.es \\ Lucía Morales-Sánchez \\ Departamento de Psicología, Universidad de Cádiz. \\ Concha Martínez-García \\ Departamento de Psicología Social, Evolutiva y de la Educación, Universidad de Huelva. \\ Paloma Gil-Olarte \\ Departamento de Psicología, Universidad de Cádiz. \\ Instituto Universitario de Investigación para el Desarrollo Social Sostenible (INDESS), Universidad de Cádiz.
}

Fecha de Recepción: 2 Marzo 2018

Fecha de Admisión: 10 Abril 2018

\begin{abstract}
RESUMEN
Un creciente cuerpo de investigación sugiere que un mayor rasgo de Inteligencia Emocional (IE) influye en el desarrollo óptimo del bienestar y el ajuste psicológico, como por ejemplo en un estado afectivo positivo. Sin embargo, hay pocos estudios que relacionen estas variables en función de las diferencias de género. Por ello, los objetivos de este trabajo son establecer si existen diferencias según el sexo en cada dimensión de la IE y en los estados afectivos. Además, este estudio se centra en comprobar la existencia de relaciones entre la IE y el afecto, estableciendo la contribución de la IE predictor del estado afectivo tanto para la muestra total como diferenciando en hombres y en mujeres. Participaron 467 estudiantes universitarios, 82,4\% mujeres, con una edad media de 21,79 años (D.T. $=5,19)$. Se utilizó para medir la IE el cuestionario TMMS-24 y para los estados afectivos el PANAS. Los resultados mostraron diferencias estadísticamente significativas $(p<.05)$ entre hombre y mujeres en el afecto positivo, siendo mayor en los hombres. Además, existen correlaciones estadísticamente significativas entre las dimensiones de la inteligencia emocional y los afectos $(p<.01)$. En cuanto al valor predictivo de la IE, los resultados mostraron que las tres dimensiones de IE predicen en ambos sexos el afecto negativo, siendo la varianza explicada de un $21,2 \%$ en mujeres y aproximadamente el doble en hombres (37,5\%). Sin embargo, con el afecto positivo son las variables atención y regulación emocional las que predicen un 16,7\% de la varianza en las mujeres,
\end{abstract}




\section{VALOR PREDICTIVO DE LA INTELIGENCIA EMOCIONAL RASGO EN LOS ESTADOS AFECTIVOS: UNA COMPARACIÓN DE GÉNERO}

dejando fuera de la ecuación a los hombres. Se concluye la existencia de diferencias de género no tanto en las dimensiones sino en el papel explicativo de la inteligencia emocional sobre el afecto, por lo que ha de tenerse en cuenta de cara a futuras intervenciones.

Palabras claves: inteligencia emocional; afecto positivo; afecto negativo; género; estudiantes

\section{ABSTRACT}

Predictive value of trait emotional intelligence in affective states: A gender comparison.

A growing body of research suggests that higher levels of Trait Emotional Intelligence (EI) influence an optimal well-being development and psychological adjustment, such as positive affective states. However, few studies relate these variables considering gender differences. Therefore, the aims of this work are to establish if there are differences according to sex, in each dimension of EI and affective states. Moreover, this study aims to check the existence of relationships between El and affect, showing the El contribution as a predictor of affective state, for both, the total sample, and differentiating between men and women. Participants were 467 university students, $82.4 \%$ women, with an average age of 21.79 years $(S D=5.19)$. The TMMS-24 questionnaire was used to measure IE, and affective states were assessed with PANAS. The results showed statistically significant differences $(p<.05)$ between men and women in positive affect, being higher for men. In addition, there were statistically significant correlations between El dimensions and affects $(p<.01)$. Regarding the predictive value of El, the results showed that the three El dimensions predicted negative affect in both sexes, with an explained variance of $21.2 \%$ in women and approximately the double in men (37.5\%). However, attention and emotional regulation are the variables that predict $16.7 \%$ of the variance of positive affect in women, leaving out of the equation to men. The existence of gender differences is demonstrated, not so much in El dimensions but in the explanatory role of El in affect, so it has to be taken into consideration in the face of future interventions.

Keywords: emotional intelligence; positive affect; negative affect; gender; students

\section{INTRODUCCIÓN}

El término Inteligencia Emocional (IE) fue acuñado por Salovey y Mayer (1990). Estos autores postulan la estructura de la inteligencia emocional como un modelo de cuatro ramas o habilidades interrelacionadas y que son definidas como "la habilidad para percibir, valorar y expresar emociones con exactitud, la habilidad para acceder y/o generar sentimientos que faciliten el pensamiento; la habilidad para comprender emociones y el conocimiento emocional y la habilidad para regular las emociones promoviendo un crecimiento emocional e intelectual" (Mayer y Salovey, 1997, p. 5).

Desde este primer modelo han surgido otros que intentan conceptualizar la IE desde diferentes perspectivas (Mestre y Fernández-Berrocal, 2007). A nivel general, se pueden clasificar según sean modelos de habilidades y modelos mixtos 0 de los rasgos. Los primeros entienden la inteligencia emocional como la capacidad de procesar la información emocional y cómo ésta mejora y guía los pensamientos. Mientras que los modelos mixtos o de los rasgos entienden la inteligencia emocional como rasgos estables de personalidad, tendencias conductuales y habilidades autopercibidas (Petrides, 2010).

Para poder evaluar el constructo de IE han surgido a lo largo de los años diferentes métodos. Entre los más utilizados encontramos los cuestionarios de auto-informe, las medidas de ejecución y evaluaciones por observadores $0360^{\circ}$ (Extremera, Fernández-Berrocal, Mestre y Guil, 2004; Mestre y Guil, 2006).

Específicamente entre los instrumentos de auto-informe, el más utilizado ha sido el Trait MetaMood Scale desarrollado por Salovey, Mayer, Goldman, Turvey y Palfai (1995). Es una escala rasgo de metaconocimiento y con ella se obtiene un índice de IE percibida, ya que nos informa de la per- 
cepción que tienen los sujetos sobre sus habilidades para atender, clarificar y reparar los propios estados emocionales.

Una gran cantidad de estudios previos han determinado que los individuos con una adecuada percepción del manejo de sus emociones presentan un desarrollo óptimo del bienestar y un buen ajuste psicológico (Fernández-Berrocal, Berrios-Martos, Extremera y Augusto, 2012; Martins, Ramalho y Morin, 2010; Mestre Navas, Gutiérrez, Guerrero y Guil, 2017; Petrides, Pita y Kokkinaki, 2007; Salguero, Fernández-Berrocal, Ruiz-Aranda, Castillo y Palomera, 2011; Schutte, Malouff, Thorsteinsson, Bhullar y Rooke, 2007). Asimismo se relaciona con bajos niveles de afecto negativo y altas puntuaciones en afecto positivo (Extremera y Fernández-Berrocal, 2005; Extremera y Rey, 2016; Gohm y Clore, 2002; Palmer, Donaldson y Stough, 2002). Incluso investigaciones recientes otorgan un papel mediador a los estados afectivos entre la IE y bienestar, como la satisfacción de vida y las fortalezas personales (Extremera y Rey, 2016; Ros-Morente, Mora, Nadal, Belled y Berenguer, 2017).

Teóricamente, los estados afectivos se conceptualizan en dos dimensiones o factores independientes para determinar las experiencias emocionales. Por una parte el afecto positivo (AP) indica que un individuo se siente entusiasmado, en alerta y activo y por otro lado el afecto negativo (AN) puede reflejar fatiga, tristeza y cansancio mental y físico (Gray y Watson, 2007; Sandín et al., 1999; Watson, Clark y Tellegen, 1988). La expresión de este tipo de experiencias se consideran importantes en la prevención de la salud física, emocional y social. De tal manera que determinados trastornos, como la ansiedad y la depresión, comparten niveles elevados de afecto negativo mientras que los niveles bajos de afecto positivo se relacionan únicamente con la depresión (Sandín et al., 1999). Por este motivo, las investigaciones deben incorporar el estudio de ambas dimensiones por sus implicaciones en la prevención y reducción de ambas psicopatologías.

Por consiguiente, un aspecto a tener en cuenta son las diferencias de género, debido a que tanto las capacidades autopercibidas como los estados afectivos muestran perfiles diferentes en hombres y en mujeres. Varios estudios observan una adecuada percepción 0 atención a los sentimientos en las mujeres y una mejor regulación de las emociones en los hombres (Mestre, Guil y Lim, 2004; Molero, Álvarez y Ávila, 2010; Valadez, Borges, Ruvalcaba, Villegas y Lorenzo, 2013). Y en cuanto al afecto, las mujeres tienen mayores niveles de AN y los hombres de AP (Gómez-Maquet, 2007; Simon y Nath, 2004). Por estos motivos, Mestre y Guil (2006) proponen que los estudios sobre inteligencia emocional interpreten las puntuaciones de los hombres y mujeres por separado.

En consecuencia, resulta de interés desde el marco de la Psicología Positiva conocer qué dimensiones de la inteligencia emocional predicen un menor afecto negativo y mayor afecto positivo en función del sexo, con el objetivo de potenciar a nivel preventivo el bienestar psicológico, la felicidad y experiencias positivas.

De manera que los objetivos de este trabajo son: a) establecer si existen diferencias estadísticamente significativas según el sexo en cada dimensión de la inteligencia emocional y en los estados afectivos; b) comprobar la existencia de relaciones entre la inteligencia emocional y el afecto positivo y negativo y c) establecer la contribución de la inteligencia emocional como predictor del estado afectivo tanto para la muestra total como en hombre y en mujeres.

\section{METODOLOGÍA}

\section{Muestra}

La muestra está compuesta por 467 estudiantes universitarios de la Universidad de Huelva, $82,4 \%(N=385)$ son mujeres y $17,6 \%(N=82)$ hombres, con una edad media de 21,79 años $(D . T$. $=5,19)$. 


\section{Instrumentos}

Trait Meta-Mood Scale (TMMS-24). La Inteligencia emocional rasgo se evaluó con la versión en español de la Trait Meta-Mood Scale (Fernández-Berrocal, Extremera y Ramos, 2004). Incluye tres dimensiones, con 8 ítems cada una, que son la Atención o Percepción (identificar las emociones propias y de los demás y saber expresarlas), la Claridad (comprensión de las emociones) y la Reparación o Regulación (capacidad de manejar las emociones). Evaluadas con respuestas tipo Likert de 5 puntos. Esta prueba, según los autores, presenta una consistencia interna de $\alpha=.90$ para percepción, $\alpha=.90$ en comprensión y $\alpha=.86$ para regulación 0 reparación emocional. En nuestra caso, la fiabilidad de la dimensión atención es de $\alpha=.88$, para la claridad de $\alpha=.90$ y para la reparación de $\alpha=.87$.

Escala de Afecto Positivo y Negativo (PANAS). Los estados afectivos se midieron a través de la versión en español (Sandín et al., 1999) según la prueba original desarrollada por Watson et al. (1988). Compuesta por 20 ítems, 10 para para el Afecto Negativo y 10 para el Afecto Positivo. Presenta una estructura bidimensional y con una valoración tipo Likert de 5 opciones. En nuestra caso, la fiabilidad de cada una de las escalas guarda una consistencia interna similar a la de los autores, $\alpha=.82$ para AP y para AN $\alpha=.80$.

Datos sociodemográficos. Los ítems que se incluyeron son sexo (hombre y mujer) y la edad.

\section{Procedimiento}

Se les administraron a todos los participantes los instrumentos mediante la creación de un formulario on-line. Se activó en el campus virtual de varias asignaturas cursadas en la Universidad de Huelva. Por consiguiente todos los estudiantes de esas asignaturas tuvieron acceso al cuestionario. Aunque previamente se les informó, en horario de clase, de los objetivos de la investigación y que su participación era totalmente anónima y voluntaria. Antes de comenzar a registrar sus respuestas debían aceptar el consentimiento informado. Por tanto, todos los estudiantes que completaron el cuestionario aceptaron dicho consentimiento.

\section{Análisis de datos}

Para la gestión de los datos se ha empleado la aplicación informática IBM SPSS 20.0 Statistics. Primero se ha realizado un estudio descriptivo (medias y desviaciones típicas) para la edad y para las distintas subescalas del TMMS-24 y del PANAS, tanto en la muestra total como diferenciando según el sexo. Los análisis para la obtención de diferencias de medias, en función del sexo, se ha elaborado a través de la prueba $t$ de Students. Además, se presenta el cálculo de correlaciones de Pearson entre las distintas dimensiones de las variables del estudio. Y por último, un estudio de regresión lineal mediante el método pasos sucesivos, con el objetivo de predecir qué dimensiones de la inteligencia emocional explican en mayor medida el afecto positivo y negativo, tanto para la muestra total como en hombres y mujeres. En estos análisis se emplea un intervalo de confianza del 95\% (IC 95\%) y se utiliza un nivel de significación de $p<.05$.

\section{RESULTADOS}

\section{Estadísticos descriptivos y diferencias de sexo}

En la Tabla 1 se puede observar las medias y desviaciones típicas de las variables del estudio, tanto para la muestra total como para los hombres y las mujeres. En general, la puntuación más alta para la IE se presenta en la dimensión Regulación Emocional y en el PANAS en el Afecto Positivo. 
Tabla 1.

Estadísticos descriptivos de las variables del estudio y análisis de las diferencias de medias según el sexo (Prueba t de Student).

\begin{tabular}{|c|c|c|c|c|c|c|c|c|c|c|}
\hline & & \multicolumn{2}{|c|}{ Total } & \multicolumn{2}{|c|}{ Mujeres } & \multicolumn{2}{|c|}{ Hombres } & \multirow[b]{2}{*}{$t$} & \multirow[b]{2}{*}{$g l$} & \multirow[b]{2}{*}{$p$} \\
\hline & & $M$ & D.T. & $M$ & D.T. & $M$ & D.T & & & \\
\hline \multirow{4}{*}{$\begin{array}{l}\text { TMMS- } \\
24\end{array}$} & idad & 21,79 & 5,19 & 21,72 & 5,36 & 22,13 & 4,34 & -.652 & 465 & .514 \\
\hline & Percepción & 29,14 & 5,21 & 29,23 & 5,27 & 28,68 & 4,91 & .870 & 465 & .385 \\
\hline & Claridad & 29,05 & 5,40 & 28,98 & 5,51 & 29,37 & 4,87 & -.581 & 465 & .562 \\
\hline & Regulación & 29,26 & 5,64 & 29,19 & 5,83 & 29,61 & 4,69 & -.794 & 139,88 & .483 \\
\hline \multirow{2}{*}{ PANAS } & $\begin{array}{c}\text { Afecto } \\
\text { negativo }\end{array}$ & 19,03 & 7,24 & 18,89 & 7,37 & 19,71 & 6,61 & -.930 & 465 & .353 \\
\hline & $\begin{array}{l}\text { Afecto } \\
\text { positivo }\end{array}$ & 29,57 & 6,60 & 29,26 & 6,71 & 31,00 & 5,87 & -2.177 & 465 & $.030^{*}$ \\
\hline
\end{tabular}

Además, se aprecia en la Tabla 1 que sólo existe una diferencia estadísticamente significativa en función del sexo para la variable Afecto Positivo, siendo mayor la puntuación en los varones.

\section{Análisis de correlación}

A continuación, Tabla 2, se puede observar las relaciones entre la edad, la inteligencia emocional y los estados afectivos. Existiendo correlaciones significativas de forma positiva entre las siguientes variables; Edad y Claridad, Percepción con Claridad, con Afecto Negativo y con Positivo, Claridad con Regulación y con Afecto Positivo, y Regulación con Afecto Positivo. Sin embargo, las correlaciones significativas de manera negativa son tanto para Claridad como para la Regulación con el Afecto Negativo. En definitiva, una mayor puntuación de las dimensiones de IE se relacionan con un mayor AP y con un menor AN, a excepción en la dimensión de Percepción o atención que se relaciona con un mayor AN.

Tabla 2.

Correlaciones de Pearson entre la edad, las dimensiones del TMMS-24 y del PANAS.

\begin{tabular}{clcccccc} 
& Variables & 1 & 2 & 3 & 4 & 5 & 6 \\
\cline { 2 - 7 } & Edad (1) & - & & & & & \\
TMMS-24 & Percepción (2) & -.045 & - & & & & \\
& Claridad (3) & $.124 * *$ & $.129 * *$ & - & & & \\
& Regulación (4) & .071 & .013 & $.308^{* *}$ & - & & \\
\multirow{2}{*}{ PANAS } & Afecto Negativo (5) & -.064 & $.303 * *$ & $-.275^{* *}$ & $-.280^{* *}$ & - \\
& Afecto Positivo (6) & .070 & $.150^{* *}$ & $.183^{* *}$ & $.350^{* *}$ & $.288^{* *}$ & - \\
\hline$* \mathrm{p}<.05 ; * * \mathrm{p}<.01 ; * * * \mathrm{p}<.001$. & & & & &
\end{tabular}

\section{Análisis de regresión}

Para establecer la contribución de la inteligencia emocional como predictor del estado afectivo se ha realizado un análisis de regresión lineal usando el método de pasos sucesivos, utilizando como variables predictoras la edad y cada una de las dimensiones de la inteligencia emocional (percepción, claridad y regulación) y como variables criterios tanto el AP como el AN. Además, se incluyó de manera diferenciada los análisis para los hombres y para las mujeres. 
En la Tabla 3 se puede apreciar los diferentes modelos, tanto para la muestra total como en hombres y mujeres, que mejor predicen el AN. Los modelos incluyen, en los tres tipos de muestras, las tres dimensiones de la IE y excluyen la variable edad.

Tabla 3.

Análisis de regresión lineal (método de pasos sucesivos) para la variable criterio Afecto Negativo tanto para muestra total como en mujeres y en hombres.

\begin{tabular}{|c|c|c|c|c|c|c|c|}
\hline & Variable criterio & $\mathrm{R}$ & $\mathrm{R}^{2}$ & R2 corregida & $\begin{array}{c}\text { Variables } \\
\text { predictoras }\end{array}$ & Beta & $t$ \\
\hline \multirow{4}{*}{ Muestra total } & Afecto Negativo & .480 & .230 & .225 & & & \\
\hline & & & & & Percepción & .338 & $8.224 * * *$ \\
\hline & & & & & Claridad & -.255 & $-5.902 * * *$ \\
\hline & & & & & Regulación & -.206 & $-4.796 * * *$ \\
\hline \multirow{4}{*}{$\begin{array}{l}\text { Muestra } \\
\text { mujeres }\end{array}$} & Afecto Negativo & .461 & .212 & .206 & & & \\
\hline & & & & & Percepción & .319 & $6.919 * * *$ \\
\hline & & & & & Claridad & -.254 & $-5.200 * * *$ \\
\hline & & & & & Regulación & -.207 & $-4.296 * * *$ \\
\hline \multirow{4}{*}{$\begin{array}{l}\text { Muestra } \\
\text { hombres }\end{array}$} & Afecto Negativo & .612 & .375 & .351 & & & \\
\hline & & & & & Percepción & .465 & $5.117 * * *$ \\
\hline & & & & & Claridad & -.261 & $-2.879 * *$ \\
\hline & & & & & Regulación & -.193 & $-2.102 *$ \\
\hline
\end{tabular}

$* \mathrm{p}<.05 ; * * \mathrm{p}<.01 ; * * * \mathrm{p}<.001$.

Los resultados del Afecto Negativo, en la muestra total, indican que las variables que explican un $23 \%$ de la varianza $(F(3,463)=46.131, p=.000)$ son las tres dimensiones de inteligencia emocional rasgo. La variable predictora que presenta un mayor peso es la Percepción, seguida de la Claridad y la Regulación (véase Tabla 3).

En las mujeres, el modelo explica un $21,2 \%(F(3,381)=34.197, p=.000)$ y en los hombres un $37,5 \%(F(3,78)=15.586, p=.000)$. En ambos análisis de regresión se presenta el mismo orden, según el peso, de las tres variables predictoras (véase Tabla 3).

Y por último, en la Tabla 4, se puede observar también los diferentes modelos que mejor predicen el AP. Quedando incluidas en los distintos análisis las dimensiones de Percepción y Regulación de la IE y excluida la variable edad y Claridad emocional. Pero en este caso, los modelos que se presentan son para la muestra total y en mujeres, dado que en hombres el análisis de regresión no introduce variables en la ecuación. 
Tabla 4.

Análisis de regresión lineal (método de pasos sucesivos) para la variable criterio Afecto Positivo tanto para muestra total como en mujeres.

\begin{tabular}{|c|c|c|c|c|c|c|c|}
\hline & Variable criterio & $\mathrm{R}$ & $\mathrm{R}^{2}$ & R2 corregida & $\begin{array}{l}\text { Variables } \\
\text { predictoras }\end{array}$ & Beta & $t$ \\
\hline \multirow{3}{*}{ Muestra total } & Afecto Positivo & .379 & .144 & .140 & & & \\
\hline & & & & & Percepción & .146 & $3.392 * *$ \\
\hline & & & & & Regulación & .348 & $8.103 * * *$ \\
\hline \multirow{3}{*}{$\begin{array}{l}\text { Muestra } \\
\text { mujeres }\end{array}$} & Afecto Positivo & .409 & .167 & .163 & & & \\
\hline & & & & & Percepción & .172 & $3.687^{* * *}$ \\
\hline & & & & & Regulación & .364 & $7.779 * * *$ \\
\hline
\end{tabular}

$* \mathrm{p}<.05 ; * * \mathrm{p}<.01 ; * * * \mathrm{p}<.001$.

El modelo explica un $14,4 \%$ de la varianza del Afecto Positivo $(F(2,464)=38.930, p=.000)$, en la muestra total, a través de las dimensiones de Percepción y Regulación de la IE. La variable predictora que presenta un mayor peso es la Regulación seguida de la Percepción (véase Tabla 4). Del mismo modo, en las mujeres, el modelo explica un $16,7 \%(F(2,382)=38.339, p=.000)$ mediante las mismas variables predictoras.

\section{DISCUSIÓN}

Los resultados encontrados en esta muestra de universitarios no manifiestan diferencias de sexo sobre la percepción que tienen de sus habilidades emocionales. De tal manera, no podemos confirmar el perfil encontrado en otros estudios que relacionan una mayor capacidad de atender a los sentimientos en las mujeres y una mayor regulación en los varones (Mestre et al., 2004; Molero et al., 2010; Valadez et al. 2013). Sin embargo, si está en consonancia con otros trabajos donde no se muestran diferencias de género (Cazalla-Luna y Molero, 2014; Prieto-Rincón, Inciarte-Mundo, Rincón-Prieto y Bonilla, 2008). Así diversos autores apuntan a que la discordancia de resultados puede explicarse porque la evaluación de la IE se realiza con distintos instrumentos (Extremera, Fernández-Berrocal y Salovey, 2006; Fernández-Berrocal et al., 2004).

Del mismo modo, se ratifica que los hombres tienen un estado de ánimo más positivo que las mujeres, es decir, se sienten más activos y enérgicos (Gómez-Maquet, 2007; Simon y Nath, 2004). No obstante, no encontramos estas diferencias en el AN, al igual que otros estudios (Alcalá, Camacho y Giner, 2007; Robles y Páez, 2003; Sandín et al., 1999). Para explicar estas controversias en la expresión emocional en función del género debemos apreciar que pueden estar implicados multitud de factores, como por ejemplo los condicionantes sociales, culturales y personales.

En lo que respecta a la relación que se establece entre la IE y los estados afectivos, se ha observado lo esperado, o sea unas puntuaciones altas en IE relacionan con bajos niveles de afecto negativo y altas puntuaciones en afecto positivo (Extremera y Fernández-Berrocal, 2005; Extremera y Rey, 2016; Gohm y Clore, 2002; Palmer et al. 2002). Según los estudios anteriores y nuestros resultados, comprender y regular adecuadamente las emociones implica que se asocie con una mayor expresión emocional positiva, alegre y activa y menos triste, fatigada o negativa. No obstante prestar mucha atención a nuestras emociones se relaciona con un aumento de vivir las emociones de manera tanto negativa como positiva. En consecuencia, como afirma Extremera y Berrocal (2005), una persona que presta mucha atención a sus emociones pero tiene problemas a la hora de regularlas no llevará a cabo las estrategias adecuadas para un estado de ánimo efectivo. 
Nuestro último objetivo es establecer la contribución de la inteligencia emocional como predictor del estado afectivo tanto para la muestra total como en hombre y en mujeres. En primer lugar, para la muestra total, se ha observado que el AN es explicado en un $23 \%$ por las tres dimensiones de la IE y el AP en un 14,4\% por la atención y la regulación emocional. En segundo lugar, para las mujeres, aparecen en los modelos las mismas variables predictoras para ambos estados afectivos que en la muestra total, lo único que el porcentaje de explicación es inferior para la predicción del AN $(21,3 \%)$ y superior para el AP $(16,7 \%)$. Y en último lugar, es en la muestra de varones donde los rasgos de inteligencia emocional predicen en un mayor porcentaje el AN $(37,5 \%)$, en cambio las variables emocionales salen de la ecuación cuando intentamos explicar las experiencias emocionales positivas. Por consiguiente, sería necesario seguir indagando esta cuestión de manera más profunda.

En general, se presentan unos porcentajes adecuados de predicción de las dimensiones de la inteligencia emocional sobre los estados afectivos, y con ello destacar cómo estudios otorgan un papel mediador sobre diferentes variables que potencian la salud física y psicológica, como por ejemplo la satisfacción de vida, las virtudes y fortalezas humanas (Extremera y Rey, 2016; GómezRomero, Limonero, Toro, Montes-Hidalgo y Tomás-Sábado, 2017; Zeidner, Matthews y Roberts, 2012). Aún así, según nuestros resultados planteamos la necesidad de observar estas relaciones y mediaciones diferenciando en función del género.

\section{CONCLUSIONES}

Se concluye la existencia de diferencias de género no tanto en las dimensiones sino en el papel explicativo de la inteligencia emocional sobre el afecto, por lo que ha de tenerse en cuenta de cara a futuras intervenciones. Por tanto, los programas de intervención en IE se deberían diseñar con el fin de aumentar la autoeficacia de los individuos en la comprensión y regulación de sus emociones. Sin embargo, habría que estudiar si la efectividad de estos programas se producen por igual en ambos sexo.

\section{REFERENCIAS}

Alcalá, V., Camacho, M. y Giner, J. (2007). Affect and depression in the elderly. Psicothema, 19(1), 49-56.

Cazalla-Luna, N. y Molero, D. (2014). Inteligencia emocional percibida, ansiedad y afectos en estudiantes universitarios. REOP - Revista Española de Orientación y Psicopedagogía, 25(3), 56-73.

Extremera, N. y Fernández-Berrocal, P. (2005). Inteligencia emocional percibida y diferencias individuales en el meta-conocimiento de los estados emocionales: una revisión de los estudios con el TMMS. Ansiedad y estrés, 11(2-3), 101-122.

Extremera, N., Fernández-berrocal, P. y Salovey, P. (2006). Spanish version of the Mayer-saloveycaruso Emotional Intelligence Test (msceit). Version 2.0: Reliabilities, age and gender differences. Psicothema, 18, 42-48.

Extremera, N. y Rey, L. (2016). Ability emotional intelligence and life satisfaction: Positive and negative affect as mediators. Personality and Individual Differences, 102, 98-101. https://doi.org/10.1016/j.paid.2016.06.051

Extremera, N., Fernández-Berrocal, P., Mestre, J. M. y Guil, R. (2004). Medidas de evaluación de la inteligencia emocional. Revista Latinoamericana de Psicología, 36(2). Recuperado a partir de http://www.redalyc.org/resumen.oa?id=80536203

Fernández-Berrocal, P., Berrios-Martos, M. P., Extremera, N. y Augusto, J. M. (2012). Inteligencia emocional: 22 años de avances empíricos. Behavioral Psychology/Psicología Conductual, 20(1), $5-13$. 
Fernández-Berrocal, P., Extremera, N. y Ramos, N. (2004). Validity and reliability of the spanish modified version of the trait meta-mood scale. Psychological Reports, 94(3), 751-755. https://doi.org/10.2466/pr0.94.3.751-755

Gohm, C. L. y Clore, G. L. (2002). Four latent traits of emotional experience and their involvement in well-being, coping, and attributional style. Cognition \& Emotion, 16(4), 495-518.

Gómez-Maquet, Y. (2007). Cognición, emoción y sintomatología depresiva en adolescentes escolarizados. Revista Latinoamericana de Psicología, 39(3). Recuperado a partir de http://www.redalyc.org/resumen.oa?id=80539301

Gómez-Romero, M. J., Limonero, J. T., Toro, J., Montes-Hidalgo, J. y Tomás-Sábado, J. (2017). Relación entre inteligencia emocional, afecto negativo y riesgo suicida en jóvenes universitarios. Ansiedad y Estrés, 21.https://doi.org/10.1016/j.anyes.2017.10.007

Gray, E. K. y Watson, D. (2007). Assessing positive and negative affect via self-report. En J. A. Coan y J. J. B. Allen. Handbook of emotion elicitation and assessment, (pp. 171-183). New York: Oxford University Press.

Martins, A., Ramalho, N. y Morin, E. (2010). A comprehensive meta-analysis of the relationship between Emotional Intelligence and health. Personality and Individual Differences, 49(6), 554564. https://doi.org/10.1016/j.paid.2010.05.029

Mayer, J. D. y Salovey, P. (1997). What is emotional intelligence? En P. Salovey y D. Sluyter. Emotional Development and Emotional Intelligence: Educational Implications, 2nd edn (pp. 331). New York: Basic.

Mestre, J. M. y Fernández-Berrocal, P. (2007). Manual de inteligencia emocional. Madrid: Pirámide.

Mestre, J. M., Guil, R. G. y Lim, N. (2004). Inteligencia emocional: ¿a qué pueden deberse las diferencias respecto a la variable género? En E. Barberá. Motivos, emociones y procesos representacionales: de la teoría a la práctica (pp. 313-324). Valencia: Fundación universidad-empresa de valencia.

Mestre, J. y Guil, R. (2006). Medidas de ejecución versus medidas de autoinformes de inteligencia emocional. Ansiedad y estrés, 12(2-3), 413-425.

Mestre, J. M., Gutiérrez, J. M., Guerrero, C. y Guil, R. (2017). Gestión de emociones en el día a día. Madrid: Ediciones Pirámide.

Molero, D., Álvarez, F. O. y Ávila, M. R (2010). Diferencias en la adquisición de competencias emocionales en función del género. Revista Electrónica de Investigación y Docencia (REID), O(3). Recuperado a partir de https://revistaselectronicas.ujaen.es/index.php/reid/article/view/1167

Palmer, B., Donaldson, C. y Stough, C. (2002). Emotional intelligence and life satisfaction. Personality and individual differences, 33(7), 1091-1100.

Petrides, K. V., Pita, R. y Kokkinaki, F. (2007). The location of trait emotional intelligence in personality factor space. British Journal of Psychology, 98(Pt 2), 273-289. https://doi.org/10.1348/000712606X120618

Petrides K.V. (2010). Trait Emotional Intelligence Theory. Industrial and Organizational Psychology, 3(2), 136-139. https://doi.org/10.1111/j.1754-9434.2010.01213.x

Prieto-Rincón, D., Inciarte-Mundo, J., Rincón-Prieto, C. y Bonilla, E. (2008). Estudio del coeficiente emocional en estudiantes de medicina. Revista chilena de neuro-psiquiatría, 46(1), 10-15. https://doi.org/10.4067/S0717-92272008000100002

Robles, R. y Páez, F. (2003). Estudio sobre la traducción al español y las propiedades psicométricas de las escalas de afecto positivo y negativo (panas). Salud mental, 26(1),69-75.

Ros-Morente, A., Mora, C. A., Nadal, C. T., Belled, A. B. y Berenguer, N. J. (2017). An examination of the relationship between emotional intelligence, positive affect and character strengths and 
virtues. Anales de Psicología / Annals of Psychology, 34(1), 63-67. https://doi.org/10.6018/analesps.34.1.262891

Salguero, J. M., Fernández-Berrocal, P., Ruiz-Aranda, D., Castillo, R. y Palomera, R. (2011). Inteligencia emocional y ajuste psicosocial en la adolescencia: El papel de la percepción emocional. European Journal of Education and Psychology, 4(2), 143-152.

Salovey, P. y Mayer, J. D. (1990). Emotional Intelligence. Imagination, Cognition and Personality, 9(3), 185-211. https://doi.org/10.2190/DUGG-P24E-52WK-6CDG

Salovey, P., Mayer, J. D., Goldman, S. L., Turvey, C. y Palfai, T. P. (1995). Emotional attention, clarity, and repair: Exploring emotional intelligence using the Trait Meta-Mood Scale. En J. W. Pennerbarker . Emotion, disclosure, and health (pp. 125-154). Washington, D. C.: American Psychological Assn.

Sandín, B., Chorot, P., Lostao, L., Joiner, T. E., Santed, M. y Valiente, R. M. (1999). Escalas PANAS de afecto positivo y negativo: validación factorial y convergencia transcultural. Psicothema, 11(1), 37-51.

Schutte, N. S., Malouff, J. M., Thorsteinsson, E. B., Bhullar, N. y Rooke, S. E. (2007). A meta-anaIytic investigation of the relationship between emotional intelligence and health. Personality and Individual Differences, 42(6), 921-933. https://doi.org/10.1016/j.paid.2006.09.003

Simon, R. W. y Nath, L. E. (2004). Gender and emotion in the United States: Do men and women differ in self-reports of feelings and expressive behavior? American journal of sociology, 109(5), 1137-1176.

Valadez, M. D., Borges, M. Á., Ruvalcaba, N., Villegas, K. y Lorenzo, M. (2013). Emotional intelligence and its relationship with gender, academic performance and intellectual abilities of undergraduates. Electronic Journal of Research in Educational Psychology, v11(n2), 395-412.

Watson, D., Clark, L. A. y Tellegen, A. (1988). Development and validation of brief measures of positive and negative affect: the PANAS scales. Journal of Personality and Social Psychology, 54(6), 1063-1070.

Zeidner, M., Matthews, G. y Roberts, R. D. (2012). The Emotional Intelligence, Health, and WellBeing Nexus: What Have We Learned and What Have We Missed? Applied Psychology: Health and Well-Being, 4(1), 1-30. 